\title{
Comparison of Collocation Extraction Measures for Document Indexing
}

\author{
Sasa Petrovic, Jan Snajder, Bojana Dalbelo Basic, Mladen Kolar \\ Faculty of Electrical Engineering and Computing, University of Zagreb, Croatia
}

\begin{abstract}
Automatic extraction of collocations from a corpus is a well-known problem in the field of natural language processing. It is typically carried out by employing some kind of a statistical measure that indicates whether or not two words occur together more often than by chance. As there is an aboundance of these measures proposed by various authors, we have compared some of them on a task of extracting collocations from a corpus of Croatian legal documents for the purpose of document indexing. We propose and evaluate extensions of these measures for collocations consisting of three words.
\end{abstract}

Keywords: corpus statistics, collocation extraction, statistical natural language processing, document indexing.

\section{Introduction}

There is no widely accepted definition of a collocation in the field of computational linguistics. Definitions range from identifying collocations with idioms, to saying that a collocation is just a set of words occuring together more often than by chance.

We set out to extract two types of collocations. The first type coincides with the definition of an open compound in [10]. An open compound is defined as an uninterrupted sequence of words that generally function as a single constituent in a sentence (i.e. stock market, foreign exchange, etc.). The second type of collocation we wanted to extract was less idiomatic and more compositional than an open compound, and it involved sequences of words often occuring together, interrupted by a preposition or a conjunction, and describing similar concepts (e.g. cure for cancer, guns and ammunition, etc.).
There are many possible applications for collocation extraction [7]: finding multiple word combinations in text for indexing purposes in information retrieval, automatic language generation, word sense disambiguation in multilingual lexicography, improving text categorisation systems, etc.

The purpose of the whole process of extracting collocations was, in our case, improvement of the document indexing system CADIS [6]. We believe that the definition of a collocation we adopted here will be useful for indexing purposes. For the same reason, we include some types of trigrams that are not open compounds that particular type of trigrams was found very useful for indexing performed by human experts. Focus of our work was to filter out noncollocations that could not otherwise be filtered out by POS tags and frequency alone. In order not to reduce the performance of an indexing system, we aim at high recall (near $100 \%$ ). That is why we will use the $F_{1}$ measure only for comparison of association measures, but not for actually distinguishing collocations from noncollocations.

In the following section we give more insight into related work on this topic, after which, in Section 3, a formal approach to corpus preprocessing is described. Section 4 gives a brief introduction to the used measures and their possible extensions for trigrams. In Section 5 we describe our approach to evaluating measures in more detail, while Section 6 gives and discusses the results. 


\section{Related Work}

There are a lot of papers that deal with the problem of collocation extraction, but the lack of a widely accepted definition of a collocation leads to a great diversity in used measures and evaluation techniques, depending on the purpose of collocation extraction. Smadja [10] uses collocation extraction for the purpose of language generation, so he seeks to capture longer collocations and especially idioms in order to improve his system. He uses a lot of statistical data (word frequencies, deviation, distances, strength, etc.) to accomplish the task. On the other hand, Goldman [5] uses his system FipsCo for terminology extraction, so he relies on a very powerful syntactic parser. Unlike both of them, Wu [14] sets out to extract collocations from a bilingual aligned corpus, and for this he uses a number of preprocessing steps in combination with the log-likelihood ratio and a word alignment algorithm.

Also, there is no agreed upon method for evaluating collocation extraction systems, so [10] employs the skills of a professional lexicographer, while on the other hand Thanopoulos [13] uses WordNet as a golden standard. Other authors like Evert [4] use a small sample of the entire set of candidates for comparison.

\section{Corpus Preprocessing}

Collocations are extracted according to their ranking with respect to an association measure. These measures are based on raw frequencies of words and sequences of words ( $n$-grams) in corpus, obtained as follows.

\subsection{Obtaining $n$-grams}

Let $W$ be a set of words and $P$ be a set of punctuation symbols, and $W \cap P=\emptyset$. We represent the corpus $C$ as a sequence of tokens, i.e. words and punctuation symbols, of finite length $k$ :

$$
C=\left(t_{1}, t_{2}, \ldots, t_{k}\right) \in(W \cup P)^{k} .
$$

Let $W^{+}=\bigcup_{n=1}^{\infty} W^{n}$ be the set of all word sequences. An $n$-gram is a sequence of words $\left(w_{1}, w_{2}, \ldots, w_{n}\right) \in W^{+}$. From now on, as a shorthand, we write $w_{1} w_{2} \cdots w_{n}$ instead of $\left(w_{1}, w_{2} \ldots, w_{n}\right)$. Each occurence of an $n$-gram can be represented by a tuple $\left(w_{1} \cdots w_{n}, i\right) \in$ $W^{+} \times \mathbf{N}$, where $i \in \mathbf{N}$ is the position of the $n$-gram in $C$. Let $S$ be the set of all $n$-gram occurences in corpus $C$, defined as follows:

$$
\begin{aligned}
S=\{ & \left(w_{1} \cdots w_{n}, i\right) \in W^{+} \times \mathbf{N}: \\
& (i \leq k-n+1) \wedge \\
& \left.(1 \leq j \leq n)\left(w_{j}=t_{i+j-1}\right)\right\} .
\end{aligned}
$$

Note that $n$-grams from $S$ do not cross sentence boundaries set by the punctation symbols from $P$. There are exceptions to this rule: when a word and a punctuation following it form an abbreviation, then the punctuation is ignored. We preprocess the corpus $C$ to reflect this before obtaining $n$-grams.

\subsection{Lemmatisation}

Words of an $n$-gram occur in sentences in inflected forms, resulting in various forms of a single $n$-gram. In order to conflate these forms to a single $n$-gram, each word has to be lemmatised, i.e. a lemma for a given inflected form has to be found. In this work we restrict ourselves to ambiguous lemmatisation by not taking into account the context of the word. Let $l m: W \rightarrow \wp(W)$ be the lemmatisation function mapping each word into a set of ambiguous lemmas, where $\wp$ is the powerset operator. If a word $w \in W$ cannot be lemmatised for any reason, then $\operatorname{lm}(w)=w$.

Another linguistic information obtained by lemmatisation is the word's part-of-speech (POS). In this work we only consider the following four: nouns $(N)$, adjectives $(A)$, verbs $(V)$ and stop-words $(X)$. Here stop-words include prepositions and conjunctions. Let $P O S=$ $\{N, A, V, X\}$ be the set of corresponding POS tags. Let function pos : $W \rightarrow \wp(P O S)$ associate to each word a set of ambiguous POS tags. If word $w \in W$ cannot be lemmatised, then POS is unknown and we set $\operatorname{pos}(w)=P O S$. Let $P^{+} O S^{+}=\bigcup_{n=1}^{\infty} P O S^{n}$ be the set all POS tag sequences, called POS patterns. 


\subsection{Counting and POS Filtering}

Let $f: W^{+} \rightarrow \mathbf{N}_{0}$ be a function associating to each $n$-gram its frequency in the corpus $C$. It is defined as follows:

$$
\begin{aligned}
& f\left(w_{1} \cdots w_{n}\right)=\mid\left\{\left(w_{1}^{\prime} \cdots w_{n}^{\prime}, i\right) \in S:\right. \\
& \left.(1 \leq j \leq n)\left(\operatorname{lm}\left(w_{j}\right) \cap \operatorname{lm}\left(w_{j}^{\prime}\right) \neq \emptyset\right)\right\} \mid .
\end{aligned}
$$

Due to lemmatisation, the obtained frequency is insensitive to $n$-gram inflection.

Only $n$-grams of the appropriate POS patterns will be considered collocation candidates. Let $P_{P} \subset S_{f} \subset \mathrm{POS}^{+}$be the set of allowable POS patterns defining the POS filter. An $n$-gram $w_{1} w_{2} \cdots w_{n}$ is said to pass the POS filter if and only if:

$$
\operatorname{POS}_{f} \cap \prod_{j=1}^{n} \operatorname{pos}\left(w_{j}\right) \neq \emptyset,
$$

where $\Pi$ denotes the Cartesian product.

\section{Association Measures}

\subsection{Definitions for Digrams}

Association measures (AMs) are used to indicate the strength of association of two words. We will now describe four commonly used measures along with some of their properties.

Pointwise mutual information ${ }^{1}$ (PMI) [2] is a measure that comes from the field of information theory, and it measures the amount of information we have about the occurence of one word if we are provided with information about occurence of the other word. It is given by the formula:

$$
I(x, y)=\log _{2} \frac{P(x y)}{P(x) P(y)},
$$

where $x$ and $y$ are words and $P(x), P(y), P(x y)$ are probabilities of occurence of words $x, y$, and digram $x y$, respectively. Those probabilities are approximated by relative frequencies of the words or digrams in the corpus.
The Dice coefficient is defined as:

$$
\operatorname{DICE}(x, y)=\frac{2 f(x y)}{f(x)+f(y)},
$$

where $f(x), f(y), f(x y)$ are frequencies of words $x, y$ and digram $x y$, respectively. The Dice coefficient is sometimes considered superior to information theoretic measures, especially in translating by using a bilingual aligned corpus [7].

Next two measures emerge from the field of statistics. They deal with hypotesis testing, i.e. with acceptance or rejection of the nullhypotesis (in our case the null-hypotesis being "words $x$ and $y$ occur together by chance"). First of these measures is the chi-square test, defined as:

$$
\chi^{2}=\sum_{i, j} \frac{\left(O_{i j}-E_{i j}\right)^{2}}{E_{i j}},
$$

where $O_{i j}$ and $E_{i j}$ are observed and expected frequencies in a contingency table [7].

The log-likelihood ratio (LL) [9] (entropy version) is defined as:

$$
G^{2}=\sum_{i, j} O_{i j} \log \frac{O_{i j}}{E_{i j}}
$$

\subsection{Extending the Measures for Trigrams}

All existing AMs are defined for the association between two words, which, obviously, makes them inadequate for extracting collocations consisting of three words. Therefore, we need to extend the existing measures. An overview of the existing extensions of PMI is given in [12]. We tested the following formulae for PMI:

$$
\begin{aligned}
& I_{a}(x, y, z)=\log _{2} \frac{P(x y z)}{P(x) P(y) P(z)}, \\
& I_{b}(x, y, z)=\frac{I(x y, z)+I(x, y z)}{2}, \\
& I_{c}(x, y, z)=\frac{I(x, y)+I(y, z)+I(x, z)}{3} .
\end{aligned}
$$

\footnotetext{
${ }^{1}$ The definition of mutual information we used here is more common in corpus linguistic than in information theory, where the definition of average mutual information is more commonly used.
} 
Formula (5) is the natural extension of PMI for $n$-gram of any size $n[8]$, formula (6) is due to Boulis [1], and formula (7) is proposed by Tadic [12].

Along with extending the Dice coefficient in the same way as PMI was extended in formulas (6) and (7), we also tested the natural extension of the Dice coefficient for trigrams [8]:

$$
\operatorname{DICE}(x, y, z)=\frac{3 f(x y z)}{f(x)+f(y)+f(z)} \text {. }
$$

We also propose a heuristics for trigrams based on the assumption that for different types of collocations one should use different AMs. It basically consists of combining POS information with AMs as follows:

$$
H(x, y, z)= \begin{cases}2 I(x, z) & \text { if } X \in \operatorname{pos}(y) \\ I_{a}(x, y, z) & \text { otherwise }\end{cases}
$$

If the second word in the trigram is a stop-word (i.e. POS tag is $X$ ), we only compute the strength of association between the other two words, otherwise we compute the strength of association among all three words.

\section{Evaluating AMs}

\subsection{Corpus Preprocessing}

The corpus we used for obtaining $n$-grams and their frequencies and for testing of AMs consists of 7008 Croatian legal documents from the Croatian National Corpus [3]. It contains over 1 million words, 167911 lemmas, 1816121 digrams, and 4656013 trigrams.

For lemmatising Croatian, we used a morphological lexicon constructed by rule-based automatic acquisition [11]. The so obtained lexicon is not perfectly accurate, thus prone to lemmatisation and POS tagging errors. The POS filters used for digrams are $\mathrm{AN}$ and $\mathrm{NN}$, while for trigrams the following filters were used: ANN, AAN, NAN, NNN, NXN. Note that, as said in 3.2 , the words not found in the dictionary are given all possible POS tags.

\subsection{Our Approach to Evaluation}

Comparison of AMs is usually done by having an expert evaluate n-best candidates for each measure, and manually assign each $n$-gram a label indicating whether it is a collocation or not. This is a time consuming procedure, and it can be very tiresome for a human expert. When we take into account also the size of our corpus and the number of measures we want to compare, it becomes clear that such a comparison is impossible.

Therefore, we adopted the approach used by Evert [4] and extracted a small random sample of positive and negative examples (i.e. collocations and non-collocations), which we used to compute the precision and recall among n-best candidates for each measure. The positive examples were extracted by having a human expert read randomly selected documents and extract obvious collocations from them (e.g. martial art, organized crime, etc.). In other words, we extracted the positive examples before applying the POS and frequency filters, rather than after like in [4]. This was done so we could also compare the effect POS and frequency filtering have on the recall, i.e. how many mistakes occur due to lemmatisation and how many collocations will be lost by applying the frequency filter. The negative examples were extracted by having a human expert isolate the obvious non-collocations from a list of collocations that passed a certain POS filter (e.g. different schedule, every person, etc.). This means they were extracted after applying the POS filter because if we did that before the filtering, we would get a lot of negative examples that do not pass the POS filter, resulting in an unrealistically high precision (which would be due to a good filter, not a good measure). The random sample for digrams consists of 229 collocations (considered positive examples) and 229 non-collocations (considered negative examples), and for trigrams it consists of 100 collocations and 100 non-collocations. This, of course, does not reflect the true state of the whole population, as there are naturally more negative than positive examples. But, it does give us a solid basis to compare our measures, as one would normally expect that the relative performance of measures is independent of the test sample. 


\section{Results}

\subsection{Digrams}

The results for digrams are shown in Figure 1. They were obtained after applying POS filter and frequency filter with a threshold of 3 , meaning that a digram has to appear at least 3 times in the corpus to pass the frequency filter. Out of all digrams in the corpus, $49.5 \%$ passed the POS filter, $31.6 \%$ passed the frequency filter, and $14.1 \%$ passed both filters. We used both filters because the maximum recall for all digrams that pass these filters is $95 \%$, and we decided to tolerate a loss of about $5 \%$ of collocations. The loss of $5 \%$ of collocations is due to POS tagging errors (e.g. a NN collocation with one of the nouns incorrectly tagged as a verb does not pass the POS filter), and to the fact that there are collocations appearing less than 3 times in the corpus. From Figure 1 it is obvious that all the tested measures perform better than sorting by raw frequency (which justifies the use of AMs) and that PMI performs best, followed by chi-square and LL, while the Dice coefficient performs worst.

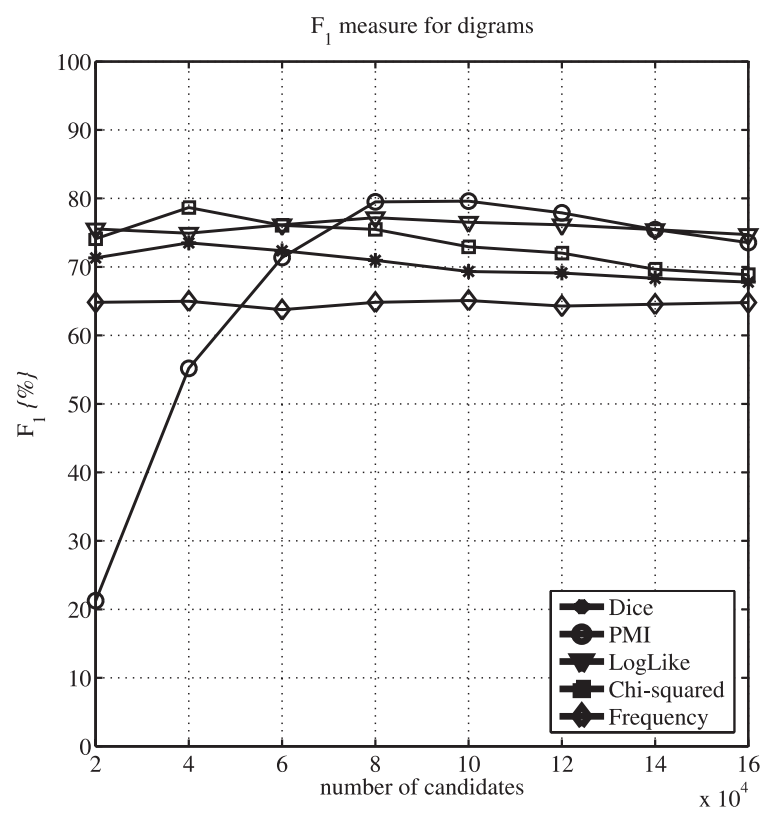

Fig. 1. $F_{1}$ Measure for digrams.

\subsection{Trigrams}

We have tested the formulæ for PMI and Dice coefficient given in Section 4, as well as the ex- tensions of chi-square and LL measures. These extensions are obtained from (6) and (7) by replacing PMI with chi-square and LL, respectively. We will, however, omit the results of all but the best extension of each measure (for each measure, the maximum $F_{1}$ score of the best extension outperforms the maximum $F_{1}$ score of other extensions by $2-5 \%$ ).

Out of all trigrams in the corpus, $32.4 \%$ passed the POS filter, $19.5 \%$ passed the frequency filter (with the threshold of 3), and only $6.1 \%$ of all trigrams passed both filters. Maximum recall for trigrams that passed both filters was only $93 \%$, which, in our opinion, is unacceptable. Therefore, unlike with digrams, we decided to use only POS filtering thereby achieving a very good recall of $99 \%$.

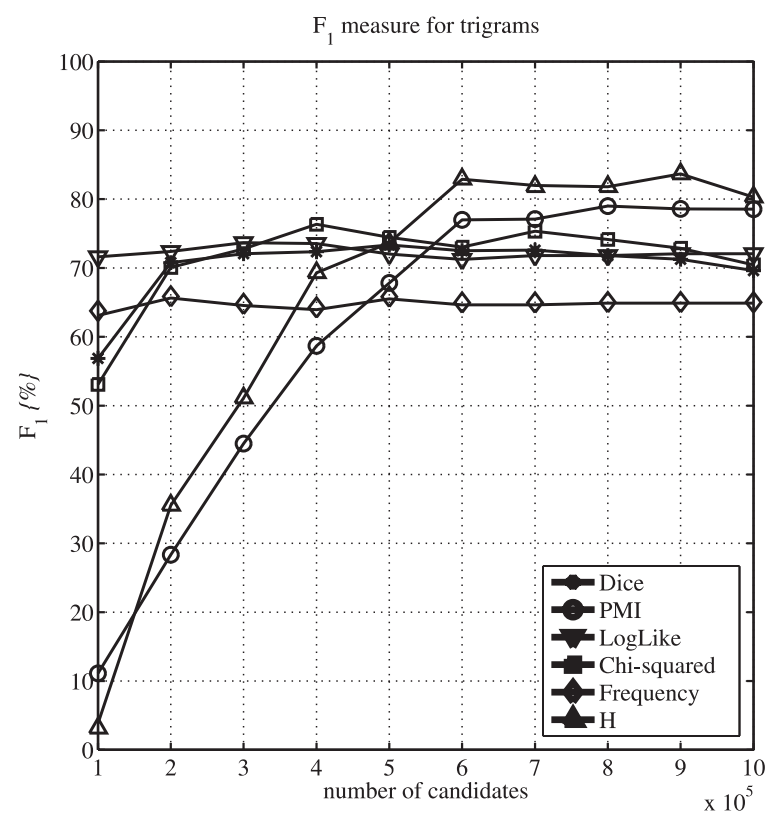

Fig. 2. $F_{1}$ Measure for trigrams.

The results for trigrams are shown in Figure 2. PMI outperformed the other three widely used measures, but the heuristics we proposed gave even better results. This confirms our intuition that different AMs should be used for extracting different types of collocations. It is also interesting to note that the best extension of LL, Dice and chi-square showed to be the one derived from (6), indicating that when extracting collocations consisting of three words, one should compute the mean between strength of association of initial/final digram with ending/starting single word. 


\subsection{Finding Relevant Collocations for Indexing Purposes}

For indexing purposes we cannot simply take the n-best candidates of the best measure as collocations, because that would lead to problems if we wished to extend our corpus (a bigger corpus obviously contains more collocations than a smaller one). On the other hand, using a threshold of an AM for distinguishing collocations from non-collocations is insensitive to corpus size. This results from the fact that a threshold of an AM tells us how strongly two (or more) words need to be associated to be considered a collocation. Obviously, that does not depend on how many other, "stronger" collocations are there in the corpus.

For example, after finding PMI to be the best choice for extracting collocations consisting of two words, we computed recall and precision for each threshold of PMI ranging from 0 to 20 (with a step of 1) and then decided that a threshold of 4 (determined by the maximum recall with the best precision) will be used to indicate if digram is a collocation or not. For trigrams, we used a threshold of 5 .

\section{Conclusion}

In this paper we compared four widely used AMs for extracting collocations consisting of two words in a corpus of Croatian legal documents. The results showed that PMI outperforms LL, chi-square and the Dice coefficient.

There are very few measures mentioned in the literature for extracting collocations consisting of three words. We therefore proposed extensions of the chi-square and LL measures in the same manner PMI and Dice were extended. Surprisingly, LL and the Dice coefficient performed similarly, while PMI again outperformed the other three tested measures. Also, we proposed a heuristics based on the assumption that for different types of collocations we should use different AMs. That heuristics gave very good results, outperforming all the tested measures.

For the actual use of collocation extraction in document indexing, one needs to find an optimal threshold of a chosen AM, and we outlined how to determine such a threshold.
For future work, we plan to experiment with other AMs and extend them for tetragrams.

\section{References}

[1] C. Boulis, Clustering of Cepstrum Coefficients Using Pairwise Mutual Information. Tehnical Report EE516, University of Washington, Seattle, 2002.

[2] K. Church, P. HANKs, Word association norms, mutual information, and lexicography. Computational linguistics, 16(1): pp. 22-29; 1990.

[3] Croatian national corpus. http://www.hnk.ffzg.hr, [01/16/2005].

[4] S. EverT, B. KRENN, Using small random samples for the manual evaluation of statistical evaluation measures. Computer speech and language, 19: pp. 450-466; 2005.

[5] J-P. Goldman, E. Wehrli, FipsCo: A syntax-based system for terminology extraction. Grammar and Natural Language Processing Conference, Universite du Quebec at Montreal, 2001.

[6] M. Kolar, I. Vukmirovic, B. Dalbelo Basic, J. SNAJDER, Computer-aided document indexing system. Journal of Computing and Information Technology, 13(4): pp. 299-305; 2005.

[7] C. Manning, H. Schütze, Foundations of statistical natural language processing. MIT Press, Cambridge, MA, USA; 1999.

[8] B. T. MCINNES, Extending the log-likelihood measure to improve collocation identification. Master thesis, University of Minnesota; 2004.

[9] M. P. OAKES, Statistics for corpus linguistics. Edinbourgh University Press, 1998.

[10] F. Smadja, K. McKeOWn, Automatically extracting and representing collocations for language generation. In Proc. of the 28th Annual Meeting of the ACL; 1990. pp. 252-259.

[11] J. SNAJDER, Rule-based automatic acquisition of large-coverage morphological lexicons for information retrieval. Tech. Report, MZOS 2003-082, ZEMRIS, FER, University of Zagreb, 2005.

[12] M. TADIC, K. SoJAT, Finding multiword term candidates in Croatian. In Proc. of IESL2003 Workshop, Borovets, Bulgaria; 2003. pp. 102-107.

[13] A. Thanopoulos, N. FAKotakis, G. KoKKInAKIS, Comparative evaluation of collocation extraction metrics. In Proc. of the LREC 2002 Conference; 2002. pp. 609-613.

[14] C-C. Wu, J. S. CHANG, Bilingual collocation extraction based on syntactic and statistical analyses. Computational Linguistics and Chinese Language Processing, 9(1): pp. 1-20; 2004. 
Received: June, 2006

Accepted: September, 2006

Contact addresses:

Sasa Petrovic

Faculty of Electrical Engineering and Computing

University of Zagreb

Unska 3, 10000 Zagreb

Croatia

Sasa.Petrovic@fer.hr

Jan Snajder

Faculty of Electrical Engineering and Computing

University of Zagreb

Unska 3, 10000 Zagreb

Croatia

Jan.Snajder@fer.hr

Bojana Dalbelo Basic

Faculty of Electrical Engineering and Computing

University of Zagreb

Unska 3, 10000 Zagreb

Bojana.Dalbelo@fer.hr

Mladen Kolar

Faculty of Electrical Engineering and Computing University of Zagreb

Unska 3, 10000 Zagreb

Croatia

Mladen.Kolar@fer.hr

SASA Petrovic is a fourth year student of computing at the Faculty of Electrical Engineering and Computing, University of Zagreb. His research interests lie in the field of artificial intelligence, in particular machine learning, text mining and natural language processing.

JAN SNAJDER received his B.S. degree in computing from the Faculty of Electrical Engineering and Computing, University of Zagreb, in 2002. $\mathrm{He}$ is currently a PhD student in the Department of Electronics, Microelectronics, Computer and Intelligent Systems at the same faculty. His research interests lie in the field of aritificial intelligence, in particular multiagent systems, machine learning, and natural language processing.

BOJANA DALBELO BASIC received her B.S. degree in mathematics from the Faculty of Sciences, University of Zagreb, in 1982 and the M.S. and $\mathrm{PhD}$. degrees in computer science from the Faculty of Electrical Engineering and Computing, University of Zagreb, in 1993 and 1997, respectively. She has published more than forty papers, mostly concerning applied multivariate statistics, fuzzy temporal reasoning and intelligent systems. Currently, she is Assistant Professor at the Faculty of Electrical Engineering and Computing, University of Zagreb. Her currrent research interests include machine learning and applications to data and text mining.

MLADEN KOLAR is a fifth year student of computing at the Faculty of Electrical Engineering and Computing, University of Zagreb. He is currently writing a thesis at the Department of Electronics, Microelectronics, Computer and Intelligent Systems at the same faculty. His research interests lie in the field of machine learning and its application on text analysis. 